\title{
Preliminary Experiments for the Assessment of V/W- band Links for Space-Earth Communications
}

\author{
James A. Nessel, Roberto J. Acosta, and Félix A. Miranda \\ Antennas and Optical Communications Branch \\ NASA Glenn Research Center \\ Cleveland, OH, USA \\ james.a.nessel@nasa.gov
}

\begin{abstract}
Since September 2012, NASA Glenn Research Center has deployed a microwave profiling radiometer at White Sands, NM, to estimate atmospheric propagation effects on communications links in the $V$ and $W$ bands $(71-86 \mathrm{GHz})$. Estimates of attenuation statistics in the millimeter wave due to gaseous and cloud components of the atmosphere show good agreement with current ITU-R models, but fail to predict link performance in the presence of moderate to heavy rain rates, due to the inherent limitations of passive radiometry. Herein, we discuss the preliminary results of these measurements and describe a design for a terrestrial link experiment to validate/refine existing rain attenuation models in the $\mathrm{V} / \mathrm{W}$ bands.
\end{abstract}

\section{INTRODUCTION}

As spectrum crowding and the need for wideband, high data rate space communications systems becomes increasingly problematic within the confines of currently utilized spectrum allocations, a typical approach is to move to higher frequencies where these limitations are not yet realized. Two such proposed communications architectures hoping to achieve multi-Gbps data rates are being investigated concomitantly by NASA, via the next generation Space Based Relay (SBR), and by AFRL, via the future military satellite communications (MILSATCOM) system, for the use of V/W-band space-earth links (NASA: 74-84 GHz, AFRL: 71-76/81-86 GHz). However, several issues related to propagation through the Earth's atmosphere must be better understood before reliable communications systems can be designed at these frequencies. These issues include: gaseous absorption, cloud attenuation, rain attenuation, scattering, scintillation, and signal depolarization. The first two components of atmospheric loss (gaseous/cloud attenuation) can be readily characterized via a passive radiometer and existing models. However, the characterization of the remaining atmospheric-induced propagation effects necessitates the use of an active transmitter/receiver link. Ideally, a space-based beacon should be employed to characterize the full propagation path, but, in the absence of such an opportunity, a terrestrial link can provide valuable information which can be used to enhance passive radiometric measurements. In this paper, preliminary results of gaseous and cloud attenuation estimates in the V/Wband derived from a microwave profiling radiometer deployed at White Sands, NM, is presented as a first-order estimate of
V/W-band communications system performance. An enhancement of this experiment is then proposed via the use of a terrestrial $\mathrm{V} / \mathrm{W}$-band link as a means to improve the full characterization of propagation losses through the atmosphere.

\section{RADIOMETRIC CHARACTERIZATION OF WHITE SANDS}

A Radiometrics MP-3000A Temperature, Humidity, and Liquid Profiler has been deployed at White Sands, NM, since September 1, 2012. The MP-3000A measures the brightness temperatures of $21 \mathrm{~K}$-band and $14 \mathrm{~V}$-band channels for the monitoring of the water vapor absorption line and oxygen absorption line, respectively. The radiometer employs a proprietary neural networking algorithm trained by local radiosonde data to derive temperature and water vapor concentration profiles up to $10 \mathrm{~km}$ through the atmosphere. This information, combined with the measured brightness temperatures across the spectrum provide the means to accurately estimate gaseous and cloud attenuation, based on the models defined in the ITU-R recommendations, as shown below in Figure 1 [1-2].

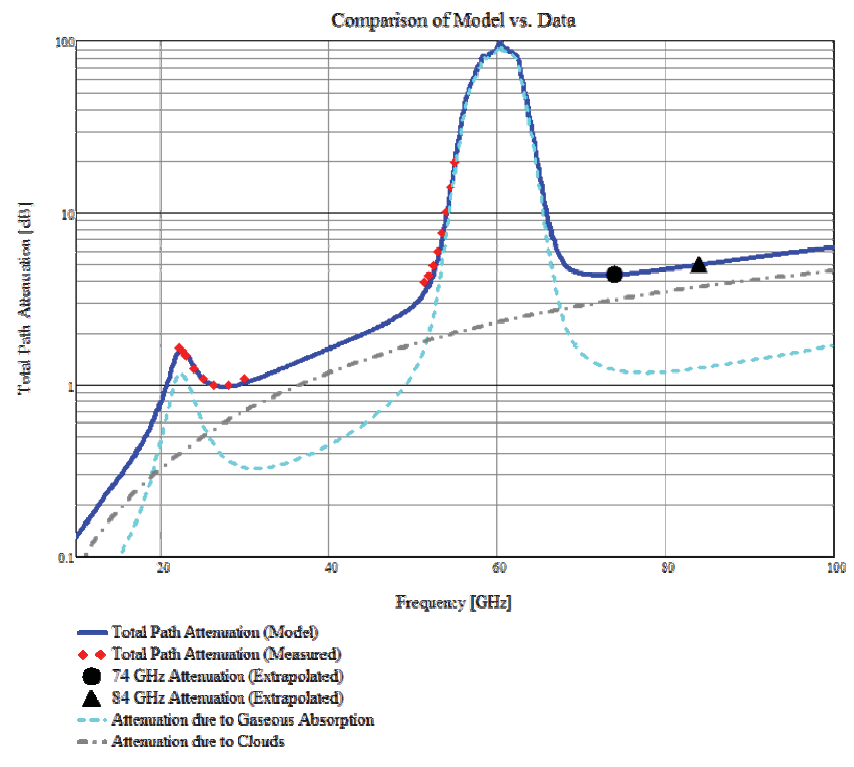

Figure 1. Gaseous and cloud attenuation curves derived from radiometer-measured sky brightness temperature and ITU-R models. 
Figure 1 shows a plot of the gaseous and cloud attenuation models, using the radiometer-derived atmospheric profiles, compared with attenuation data calculated from the radiometermeasured brightness temperatures. Figure 1 indicates how, for this particular instance in time, the presence of a cloud along the line of sight path influences the total path attenuation derived from the measurement. By fitting data collected at each instance in time to the gaseous and cloud attenuation models described in [1-2], a statistical representation of the system losses due to the atmosphere can be defined. The Cumulative Distribution Function (CDF) of losses due to gaseous and cloud attenuation for the three months of data collection in White Sands, NM, is shown in Figure 2 below.

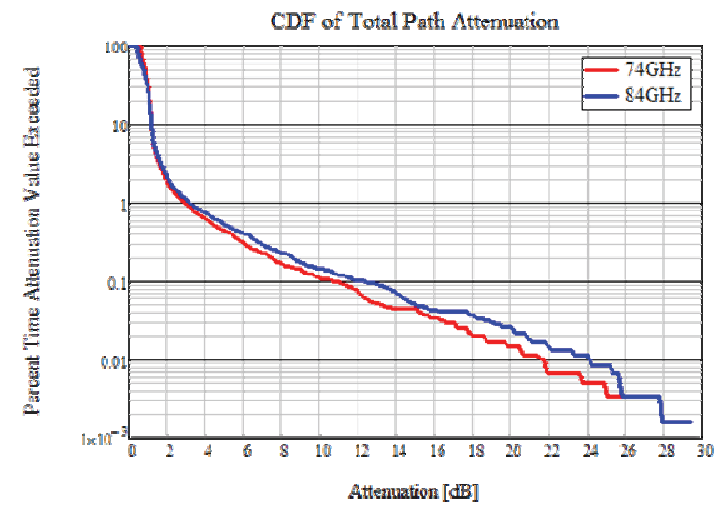

Figure 2. CDF of estimated total path attenuation at 74 and 84 $\mathrm{GHz}$ derived from radiometer data and models.

\section{Proposed Terrestrial LinK EXPERIMENT}

Gaseous absorption and cloud attenuation characterization in the V/W bands is readily measured via the use of a multispectral profiling radiometer. However, during moderate rain rates, when scattering effects begin to dominate absorption effects, a passive radiometer is incapable of accurate signal attenuation measurement. In this way, a secondary experiment is required which implements an active transmitter/receiver link to determine the contribution of these loss mechanisms in the $\mathrm{V} / \mathrm{W}$ band. One method to accomplish this, without the use of a space-based beacon, is via a terrestrial link along a slant path. A proposed architecture for the terrestrial link experiment is shown in Figure 3. From a mountain top position, a moderate elevation angle can be achieved along a slant path which can be used to characterize rain attenuation and scattering/depolarization effects. Experiment requirements are summarized in Table I, with justifications described below.

- Dual frequency K (20.2 GHz) and W (80.8 GHz) band measurement will provide information on a scaling factor which can exploit existing K-band attenuation statistics to derive long term W-band statistics [3].

- Cross-polarization isolation $>25 \mathrm{~dB}$ at the transmitter and receiver is required to effectively measure any depolarization effects, as this is the typical level realized [4].

- Dynamic range $>50 \mathrm{~dB}$ is required to characterize $\mathrm{W}$ band attenuation statistics $>99.9 \%$ of time [4].

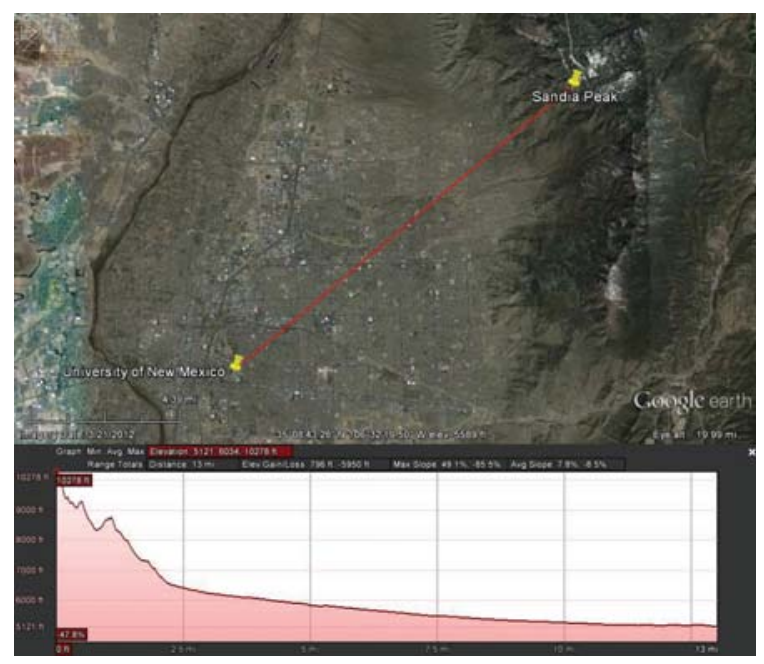

Figure 3. Top view and elevation profile for identified site in Albuquerque, NM, for terrestrial propagation experiment.

TABLE I. EXPERIMENT DESIGN SPECIFICATIONS.

\begin{tabular}{|l|c|}
\hline Experiment Parameter & Value \\
\hline Slant Path Distance & $20 \mathrm{~km}$ \\
\hline Elevation Angle & $5 \mathrm{deg}$ \\
\hline Transmitter & $20.2,80.8 \mathrm{GHz}$ \\
\hline Frequency & $0 \mathrm{dBW}$ \\
\hline EIRP & Linear (single channel) \\
\hline Polarization & $>25 \mathrm{~dB}$ \\
\hline Cross-Polarization Isolation & $1.2 \mathrm{~m}(\mathrm{Ka}), 0.6 \mathrm{~m}(\mathrm{~V})$ \\
\hline Receiver & Dual Linear \\
\hline Antenna Aperture & $<1200 \mathrm{~K}$ \\
\hline Polarization & $10 \mathrm{~Hz}$ \\
\hline System Temperature & $>50 \mathrm{~dB}$ \\
\hline Sampling Rate &
\end{tabular}

\section{CONCLUSIONS}

For three months, radiometer data have been collected at White Sands, NM, which have provided estimates of gaseous and cloud attenuation statistics in the V/W-band. However, a full characterization of $\mathrm{V} / \mathrm{W}$-band link performance requires the additional characterization of the effects of rain attenuation for space-earth communications systems to be designed reliably. Thus, a proposed terrestrial link experiment is described which can effectively accomplish this task. Through the conduction of the terrestrial link experiment, critical firstorder information on rain attenuation at $\mathrm{V} / \mathrm{W}$-band can be gathered without the need for a costly space-based beacon source. A dedicated measurement campaign for a minimum of 5 years is required to develop models for system performance at the New Mexico site.

\section{REFERENCES}

[1] ITU-R Recommendation P.676-9, "Attenuation by Atmospheric Gases," Feb. 2012.

[2] ITU-R Recommendation P.840-5, "Attenuation due to Clouds and Fog," Feb. 2012.

[3] A. Ali, et. al., "Experimental Studies of Terrestrial mm-Wave Links - A Review Part," J. Eng. Sci., Vol. 12 (2), pp.197-212, 1986.

[4] R. Acosta, et. al., "W/V-Band RF Propagation Experiment Design," $18^{\text {th }}$ $\mathrm{Ka}$ and Broadband Comm. Conference, Ottawa, Canada, Sep. 2012. 